\title{
Wakefield potentials of corrugated structures
}

\author{
A. Novokhatski ${ }^{*}$ \\ SLAC National Accelerator Laboratory, Menlo Park, California 94025, USA
}

(Received 20 August 2015; published 22 October 2015)

\begin{abstract}
A corrugated structure, which is used in "dechirper" devices, is usually a pipe or two plates with small corrugations (bumps) on the walls. There is a good single-mode description of the wake potentials excited by a relativistic bunch if the wave length of the mode is much longer than the distance between the bumps in the pipe. However, ultrashort bunches, which are now used in free electron lasers, excite much higher frequency fields and the corresponding wake potentials will be very different from the single-mode description. We have made analyses of these wake potentials based on a numerical solution of Maxwell's equations. It was confirmed that the behavior of the wakefields of ultrashort bunches in corrugated structures is not much different from the fields excited usually in accelerating structures where the wake potentials are described by the exponential function. For a practical application we present results for the SLAC "dechirper." We also carried out calculations for a similar device, that was installed and measured at the Pohang Accelerator Laboratory, Korea. We find very good agreement with the experimental results.
\end{abstract}

DOI: 10.1103/PhysRevSTAB.18.104402

PACS numbers: 41.60.-m, 29.20.Ej, 41.20.-q, 41.75.Ht

\section{INTRODUCTION}

The precise knowledge of the wakefields generated in different elements of free electron lasers (FEL) including accelerator, beam transport and undulators has become very important with increasing power and efficiency of x-ray production. Usually it is a "negative" effect. For example in Ref. [1] it is demonstrated how the wakefields generated in collimators may decrease the FEL performance. However the effect from wakefields can also be "positive" if this field is used to improve the energy spectrum. These wakefields can be generated in the accelerator (linac) or in special devices- "dechirpers." To our knowledge the word "dechirper" was first introduced in Ref. [2] where "the first experimental study was presented of a corrugated wall device that uses wake fields to remove a linear energy correlation in a relativistic electron beam." Of course different types of structures (dielectric, resistive) can be used as a dechirper, but we will analyze only corrugated wall structures in this publication.

There are several publications which have some sort of formula for the wakefields in corrugated structures. The references to these publications can be found in Refs. $[3,4]$. These formulas are derived from the assumption of a single dominating mode and the Green's function for the wake potentials is described as a damped cosine function. Although they referred to our 1997 publication [5], where we found a similarity to the wakefields in corrugated structures

"novo@slac.stanford.edu

Published by the American Physical Society under the terms of the Creative Commons Attribution 3.0 License. Further distribution of this work must maintain attribution to the author $(s)$ and the published article's title, journal citation, and DOI. with the fields in a tube with a thin dielectric layer, the authors do not fully analyze the applicability of the single mode approach to describe wakefields of very short bunches. A corrugated wall structure is planned for use in the device that causes an additional energy loss along the very short bunches of the linac coherent light source (LCLS) [4]. It now becomes very important to check how well a single mode approach can describe the fields, that are excited in corrugated structures and perhaps another description must be used. For the FEL application the dechirper has a small sized corrugation structure, of the order of millimeters. Electron bunches in the FEL have a length of a few microns. If we scale the bunch length together with the structure size up to ten times or more we will get geometry environments that are very similar to the linear accelerating structures. As we know, the wakefields in the accelerating structures are not described by a single mode. There is another description, which contains all modes, such as the Green's function for the TeV-energy superconducting linear accelerator (TESLA) accelerating structure [6] or the Stanford Linear Accelerator Center (SLAC) accelerating structure [7] described in the following equation:

$$
w_{\|}=\frac{Z_{0} c}{\pi a^{2}}\left(1+\alpha \frac{s}{s_{0}}\right) \exp \sqrt{\frac{s}{s_{0}}}
$$

In this equation $s$ is the longitudinal position in a bunch, $Z_{0}$ is the impedance of free space, $c$ is the speed of light, $a$ is the effective radius of the aperture, $\alpha$ and $s_{0}$ are fitted parameters.

We may assume that the wake potentials of a corrugated structure excited by short bunches are also described by this or a similar function. To check this assumption we calculated wake potentials of the corrugated structure using a computer code NOVO [8], which was specially designed to calculate wakefields of very short bunches. This code has 
been benchmarked based on a good agreement with the wakefields measured in the LCLS-LTU [9]. Recently the code was extended for the wakefield calculations in rectangular beam chambers [10].

\section{DECHIRPER}

As mentioned above, a dechirper takes energy from the beam through the interaction of the bunch electro-magnetic field with a metal corrugated structure. The practical design of the dechirper consists of two identical movable parallel plates (jaws) with corrugated walls in a form of the periodic set of planar ridges. A schematic drawing of the dechirper is shown in Fig. 1. The dechirper, which is planned to be installed at LCLS, has the following parameters of the corrugated structure. The period is $0.5 \mathrm{~mm}$, the thickness of a ridge is a half of a period and the transverse sizes of a ridge are: $h=0.5 \mathrm{~mm}, L x=12 \mathrm{~mm}$. Definitions of the sizes are given in Fig. 1. The total length $L$ of the corrugated structure is $2 \mathrm{~m}$. The gap between two jaws is adjustable. The nominal gap is $1.4 \mathrm{~mm}$.

We start with calculations to make a comparison with the single mode approach. We calculated wake potential of a relatively long bunch of 25 micron and for a relatively long range of distance after a bunch for a nominal gap. This wake potential is shown in Fig. 2.

The shape of the wake potential seems to be easily fitted by a damped cosine function. This approximation is shown by a red line in Fig. 2. The frequency of this mode is $105.5 \mathrm{GHz}$ or the wave length of this mode is $28.4 \mathrm{~mm}$ which is in agreement with calculations in Ref. [4]. However, as we can see from the plot, this fit does not give a good approximation at small distances: in the bunch region. For a shorter bunch this approximation can result in a much larger error. Calculated wake potentials of 10 and of 5 micron bunches are shown in Fig. 3 by magenta and green lines. We can see a large difference in the shape of the wake potentials, which have a dependence upon the distance in this region of a bunch while the single mode approach gives an almost constant value (red line). In Fig. 3 we also include a wake potential of a 25 micron bunch to show that all three wake potentials have a common part, which can

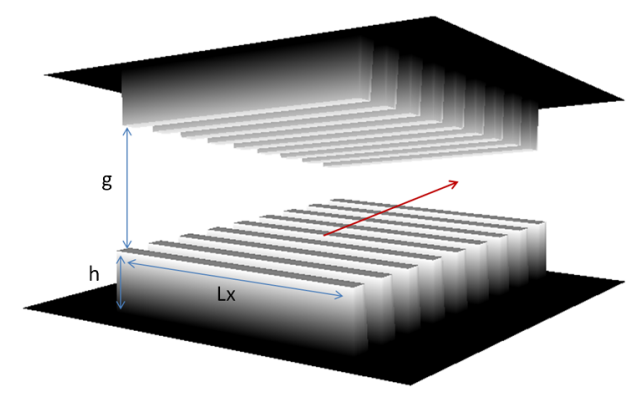

FIG. 1. A schematic drawing of a dechirper. The red line shows a bunch trajectory.

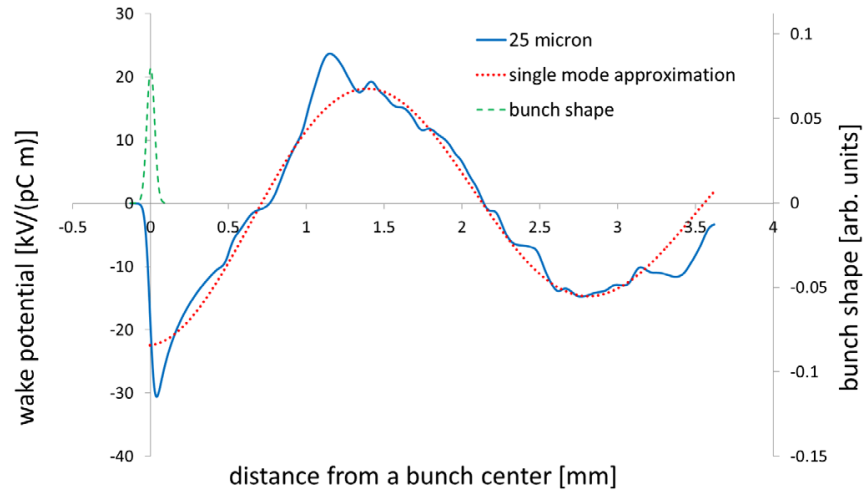

FIG. 2. Wake potential (a solid blue line) of a 25 micron bunch passing through a $2 \mathrm{~m}$ rectangular corrugated structure with a full gap of $1.4 \mathrm{~mm}$. The red dotted line shows a single mode approximation. The green dashed line shows the bunch charge distribution.

be very well approximated by an exponential function (a pink line).

Based on this approximation we suggest using the following approximation of the Green's function for corrugated structures

$$
w_{\|}(s)=A(g) \exp \sqrt{\frac{s}{s_{0}(g)}}
$$

The fitting parameters $A(g)$ and $s_{0}(g)$ are functions of the gap size $g$ (full gap). We can use this approximation as a Green's function to calculate wake potentials of bunches with any complicated charge distribution $q(s)$

$$
W(s)=\int_{-\infty}^{s} q\left(s^{\prime}\right) w_{\|}\left(s-s^{\prime}\right) d s^{\prime}
$$

We show an example of a complicated bunch shape (two horn distribution) in Fig. 4. The bunch distribution is shown by the blue line. The "head" of the bunch is on the left.

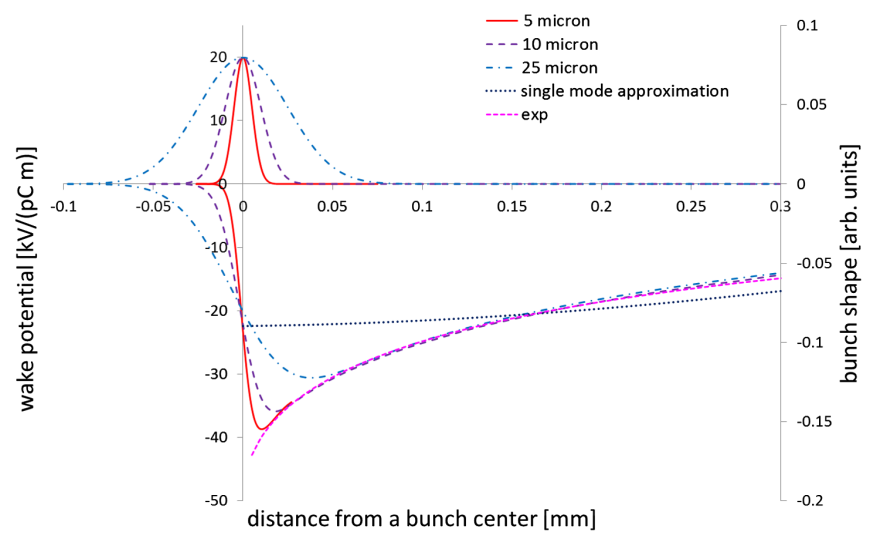

FIG. 3. Wake potential of a 5 micron bunch (solid line), 10 micron (long dashed line), and 25 micron (dash-dot line). The dotted line shows a single mode approximation and the short dashed line show the exponential function. 


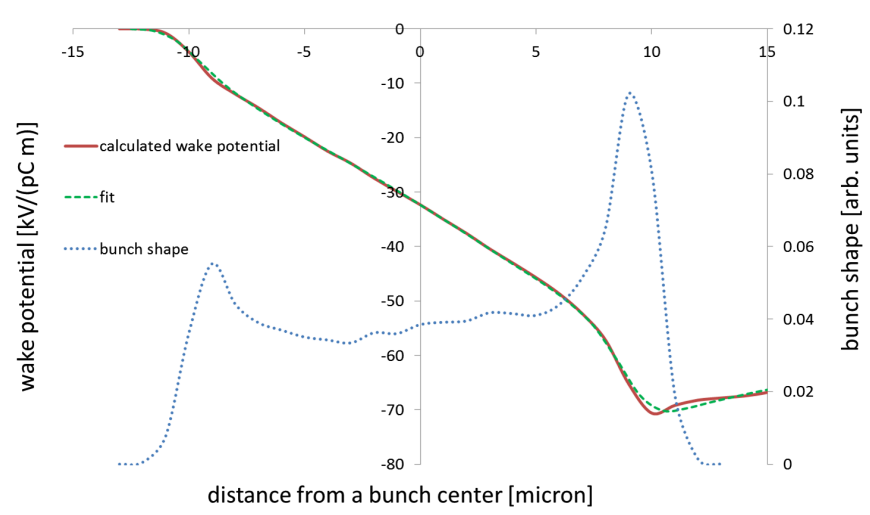

FIG. 4. Comparison of the wake potential calculated using a computer code (red solid line) and calculated using a Green's function (green dashed line) for a two horn bunch (blue dotted line) passing a dechirper with a $1 \mathrm{~mm}$ gap.

The comparison of the wake potentials calculated using a computer code (red solid line) and using a convolution with a Green's function (green dashed line) is also shown in Fig. 4. We can conclude based on this good agreement, that we can use the above mentioned Green's function (2) to calculate wakefields in the dechirper. In these calculations the gap was $1 \mathrm{~mm}$. It is interesting to note that the wake potential of a two horn bunch has two slopes. According to Fig. 4 we can compensate a possible energy chirp of the main part of a bunch, but the energy chirp of the second horn will be not compensated.

We made wakefield calculations for different gaps in order to find approximate values for $A(g)$ and $s_{0}(g)$. We have found that a more or less good approximation $( \pm 5 \%)$ for $A$ and $s_{0}$ over the range of gaps from 1 to $2 \mathrm{~mm}$ to be the following:

$$
A(g) \approx A_{0} L \frac{Z_{0} c}{g^{2}}
$$

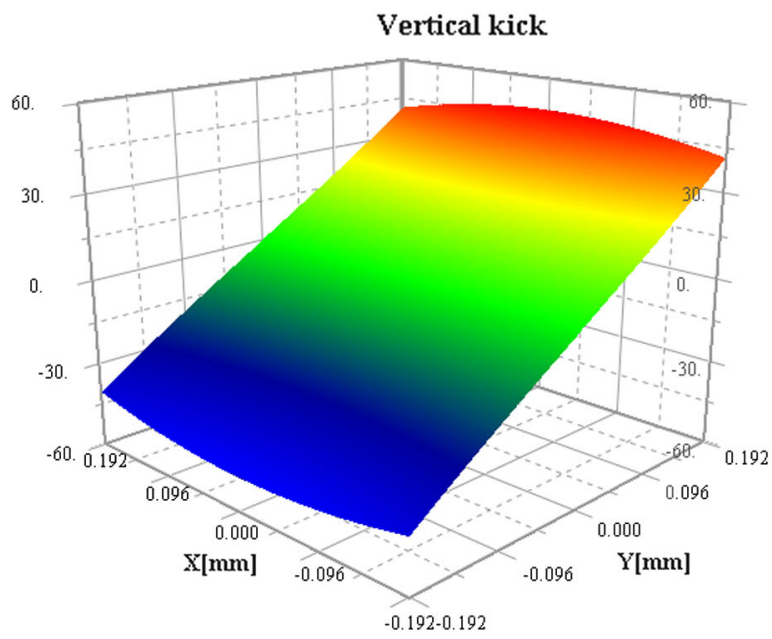

$$
s_{0}(g) \approx g \sqrt{\frac{g}{L}}
$$

with parameters: $A_{0} \approx 0.85 L \approx 55 \mathrm{~mm}$.

\section{TRANSVERSE WAKEFIELD}

The main purpose of the dechirper is to introduce energy loss along the bunch. While interacting with a longitudinal force, which is responsible for the energy loss or gain, the particles of the bunch may also interact with a transverse force. We calculate the integral of the transverse forces (transverse kick) when a bunch is in the center of the dechirper in the vertical as well as is in the horizontal direction. A two dimensional plot of the vertical and horizontal kicks is shown in Fig. 5. The orientation of the dechirper jaws is as shown in Fig. 1.

It can be seen that the dechirper acts like quadrupole lenses: defocusing in the direction of the jaws and focusing in the perpendicular direction. In general, we may represent the Green's function of the transverse kick using a Taylor expansion. In the vertical direction we have:

$w_{y}(s, y)=w_{y}^{0}\left(s, y_{1}\right)+\left.\sum_{n=1} \frac{\partial^{n}}{\partial y^{n}} w(s, y)\right|_{y=y 1}\left(y-y_{1}\right)^{n}$

When the leading particles have no offset $y_{1}=0$ and $w_{y}^{0}(s, 0)=0$ due to symmetry, then the first important term is the first derivative

$$
w_{y}(s, y)=w_{y}^{1}(s) y=\left.\frac{\partial}{\partial y} w_{y}(s, y)\right|_{y=0} y
$$

A vertical gradient of the wake fields acting on the bunch particles will be a convolution of this function and the bunch charge distribution

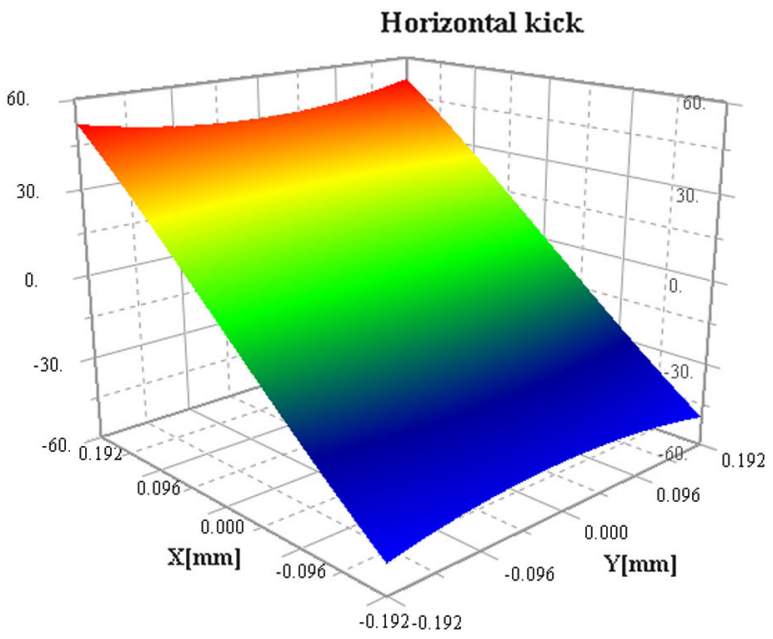

FIG. 5. Integrated vertical and horizontal force distributions when a bunch trajectory lies in the center, between the jaws and in the middle of the ridges of the dechirper $(X=0, Y=0)$. 


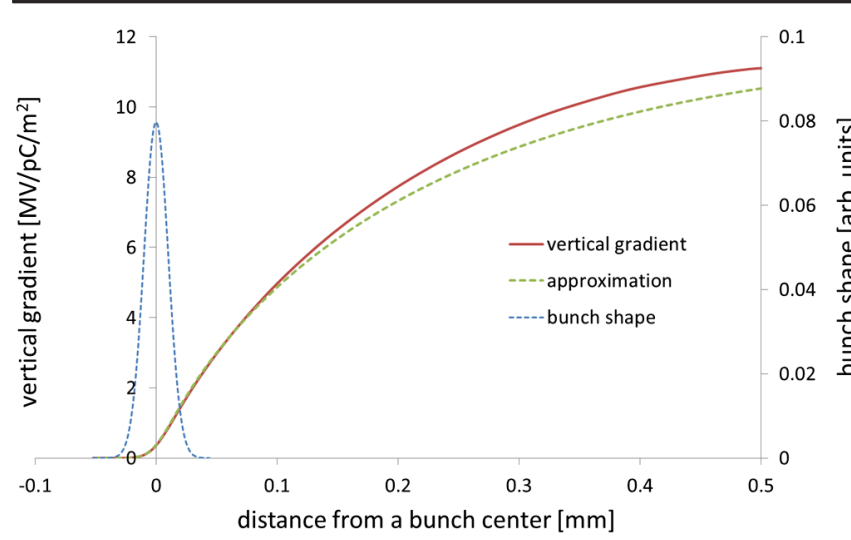

FIG. 6. Vertical gradient of the transverse wake fields of a 10 micron bunch (red line), approximation of the vertical gradient (green dashed line) and bunch, charge distribution (dotted blue line).

$$
\left.\frac{d}{d y} W(s)\right|_{y=0}=\int_{-\infty}^{s} q\left(s^{\prime}\right) w_{y}^{1}\left(s-s^{\prime}\right) d s^{\prime}
$$

We calculate this gradient for a 10 micron bunch moving through a dechirper with a gap of $1.4 \mathrm{~mm}$. Figure 6 shows this calculation.

We have found that the transverse Green's function, which defines the vertical gradient, can be approximated by the following formula:

$$
w_{y}^{1}(s) \approx \frac{1}{\Delta(g)} \frac{s}{s_{0}(g)} w_{\|}(s)
$$

The parameter $\Delta(g)$ is approximately $2.2 \mathrm{~mm}$ for gaps of 1-2 mm. One can check how well this formula describes a vertical gradient. In Fig. 6 a green dashed line shows the vertical gradient calculated using Green's function (9). We get very good agreement in the bunch region.

It was interesting to find that the Green's function for the gradient of horizontal kick has the same form like (9) but with a negative sign.

The vertical kick in the bunch is calculated in the same way using the charge distribution as in Eq. (3). Using only the first and second term of Eq. (6) we get

$$
\begin{aligned}
W_{y}(s, y) & =\int_{-\infty}^{s} q\left(s^{\prime}, y_{1}\right)\left(w_{y}^{0}\left(\tilde{s}, y_{1}\right)+w_{y}^{1}\left(\tilde{s}, y_{1}\right)\left(y-y_{1}\right)\right) d s^{\prime} \\
\tilde{s} & =s-s^{\prime}
\end{aligned}
$$

\section{A DECHIRPER AS A PASSIVE DEFLECTOR}

When a bunch travels closer to one of the jaws then the particles of the bunch get a transverse kick depending upon the position of a particle in a bunch. The tail particles get more kick. The transverse force also gets a nonlinear dependence on the transverse position. The longitudinal force also increases. As an example, in Fig. 7 we show a distribution of the transverse position of particles in the

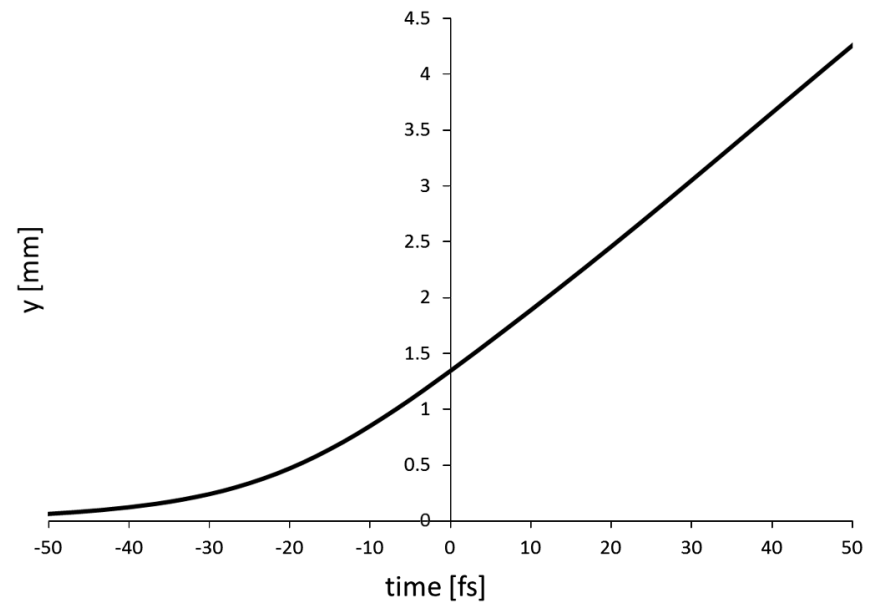

FIG. 7. The transverse position of a particle in a bunch $100 \mathrm{~m}$ downstream of the $2 \mathrm{~m}$ long dechirper. The bunch travels at a distance of $0.5 \mathrm{~mm}$ to one of the jaws. The full gap in the dechirper is $3 \mathrm{~mm}$, the bunch length is 10 micron, and the bunch charge is $100 \mathrm{pC}$.

bunch $100 \mathrm{~m}$ downstream of the dechirper. The bunch travels $0.5 \mathrm{~mm}$ closer to one of the jaws. The full gap in this case is $3 \mathrm{~mm}$, the bunch length is 10 micron and the bunch charge is $100 \mathrm{pC}$. We see that the second part of the bunch gets an almost linear kick. On an available beam screen it should be possible to see a stretching of the bunch in the deflecting direction. This effect can be used to measure the bunch distribution of very short bunches, as it was suggested in [11].

We can also use a dechirper as a "passive" deflector. The most important aspect in this proposal is that no synchronization is needed. This may help to see if a bunch has a microbunch structure due to a microbunching instability in the bunch compressors. We calculated the shape of the bunch than can be seen at the screen $100 \mathrm{~m}$ downstream of the $2 \mathrm{~m}$ long dechirper. We assume that a bunch passing off-center in the dechirper has a modulated structure (five peaks). The total bunch charge is $100 \mathrm{pC}$, the offset trajectory is $0.5 \mathrm{~mm}$ to an upper vertical jaw. Figure 8
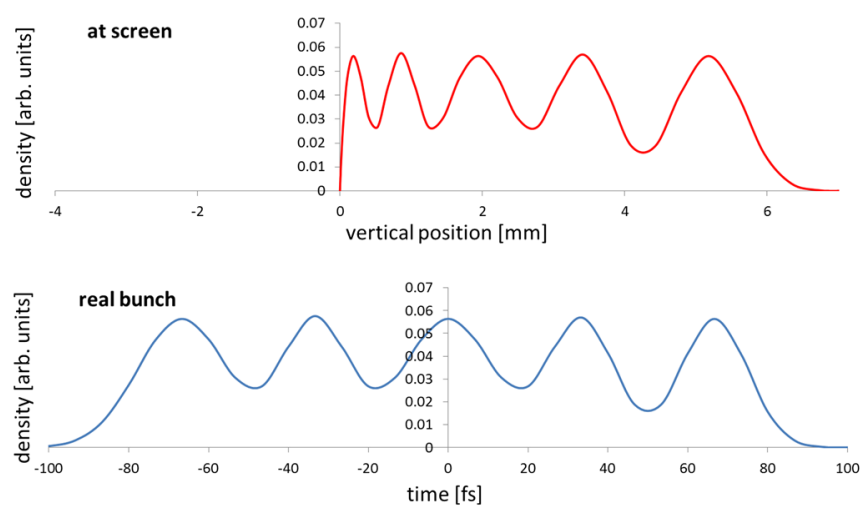

FIG. 8. Upper plot: Density distributions of a modulated bunch at the screen, $100 \mathrm{~m}$ downstream, the $2 \mathrm{~m}$ long dechirper. Lower plot: real bunch density distribution. 
shows the bunch shape at the screen (upper plot) and a real bunch distribution is shown in the lower plot. The last part of the bunch seen at the screen repeats the real bunch shape. The first part of the bunch is compressed. We can restore the first part of the bunch if we know the exact Greens function for the transverse kick analogue to the transverse gradient Green's function [Eq. (9)]. The total kick to the beam depends upon the bunch charge and the bunch length. Depending upon the resolution of the screen we may see and measure the bunch structure in atto-second range. This is another "positive" application of the wake fields.

\section{PAL-ITF EXPERIMENT}

The single mode approach was also been used for calculating the wake fields in order to make a comparison with the experimental measurement of the corrugated dechirper in Pohang Accelerator Laboratory [2]. They found a 20\% difference. They concluded the bunch charge must have been $150 \mathrm{pC}$ instead of measured $200 \mathrm{pC}$. We checked this statement even though the bunch length in the experiment was relatively large $-0.67 \mathrm{~mm}$. The geometry of the corrugated structure can be found in Ref. [2]. It has the same period of $0.5 \mathrm{~mm}$ as the SLAC dechirper, but with a little bit thicker ridges. The main difference is a larger transverse size $(L x=50 \mathrm{~mm})$, which allows using it with larger gaps.

We calculated wake potentials for an experimentally measured bunch distribution.

Figure 9 shows this distribution and corresponding wake potential for a gap of $6 \mathrm{~mm}$. It is interesting to note that again the wake potential has two slopes for a two horn bunch, so there will not be full compensation of the chirp in the dechirper. Comparing the computed wake potential, we find good agreement with the shape of the measured wake potential, which is presented in the right middle plot of Fig. 5 in Ref. [2]. We also calculated the loss factor as a function of the gap size. The results for the relative energy

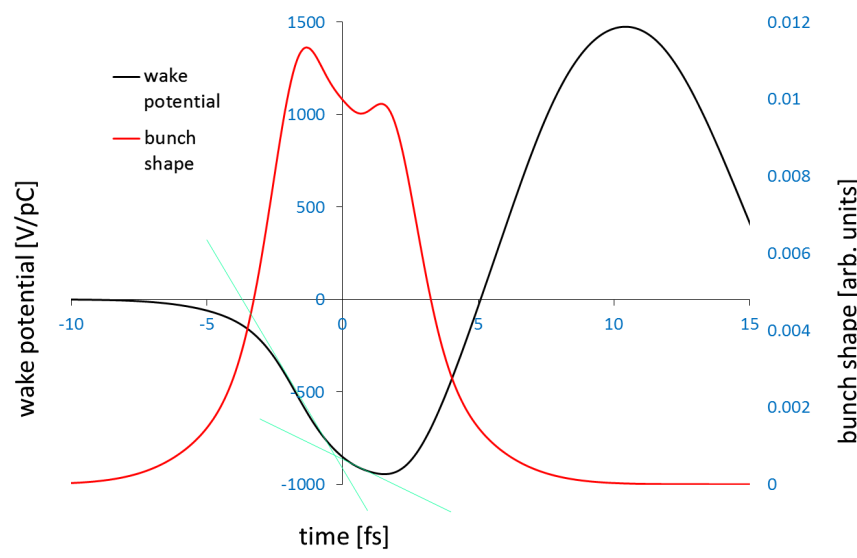

FIG. 9. The shape of the experimentally measured two horn bunch (red line) and the corresponding wake potential (black line). Light green lines show two slopes in the wake potential.

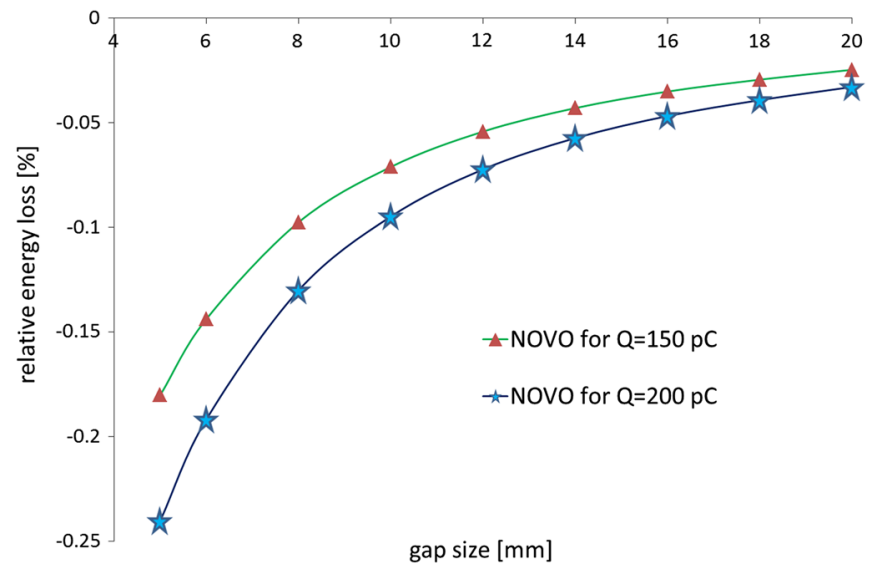

FIG. 10. Relative energy loss of a bunch with a charge of $150 \mathrm{pC}$ (green line with red triangles) and $200 \mathrm{pC}$ (black line with blue stars).

loss of a $70 \mathrm{Mev}$ bunch with a charge of $150 \mathrm{pC}$ (green line with red triangles) and $200 \mathrm{pC}$ (black line with blue stars) are shown in Fig. 10.

Comparison with a plot in Fig. 6 of Ref. [2] shows that our calculations for a $200 \mathrm{pC}$ bunch are in better agreement with the experimental measurement than for a bunch of $150 \mathrm{pC}$. We also have good agreement for the focusing effect in the horizontal direction (Fig. 11) and the vertical transverse kick (Fig. 12) for a bunch charge of 200 pC.

In these calculations we assume that the total distance to the screen (where the transverse position is measured) is $5.25 \mathrm{~m}$, which is the sum of the distance from the end of the dechirper to the screen $(4.75 \mathrm{~m})$ plus half of the dechirper length $(0.5 \mathrm{~m})$. One can compare our result with the right plot of Fig. 9 in Ref. [8] and find very good agreement. The calculated vertical position of the bunch due to an offset position of the bunch in the dechirper (red line in Fig. 12) is also in very good agreement with the measurement data (Fig. 8 in Ref. [2]). A blue line in Fig. 12 shows the energy loss of the bunch. It can be seen that the losses increase with the offset of the beam.

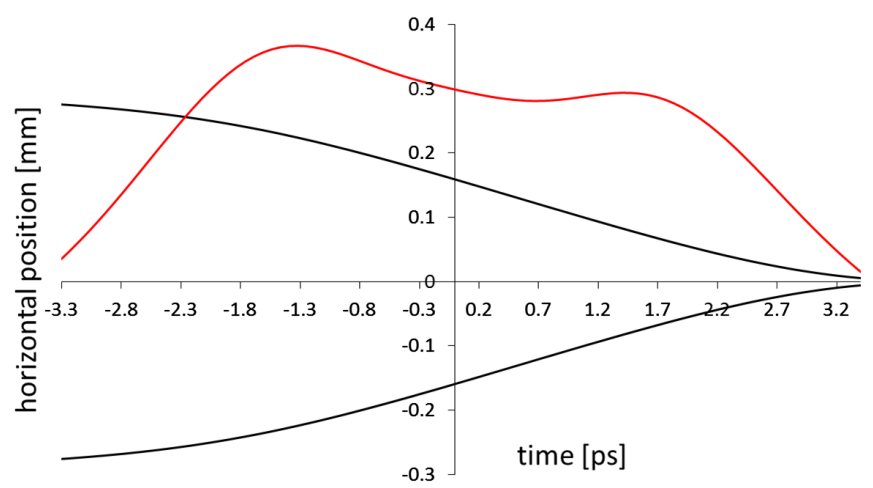

FIG. 11. Horizontal position of the bunch boundary particles (black lines) downstream of the dechirper for a bunch charge of 200 pC. The red line shows the bunch charge distribution. 


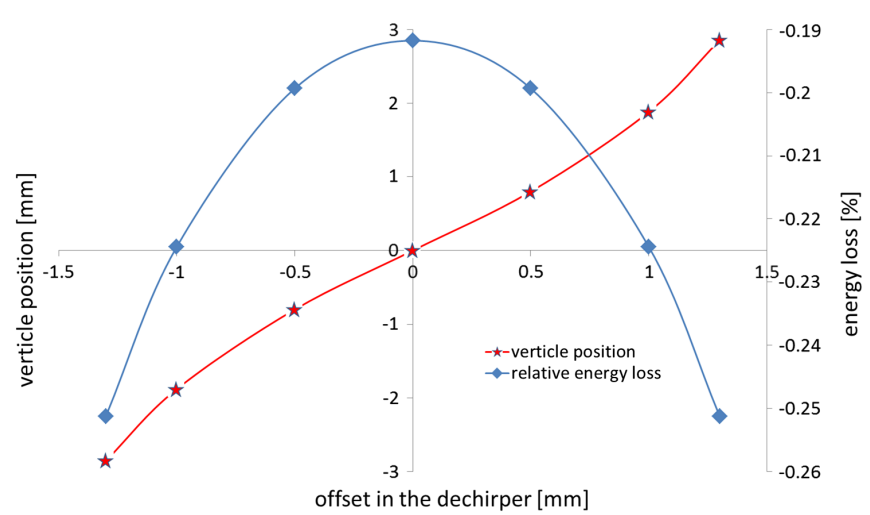

FIG. 12. Vertical position of the bunch downstream of the dechirper (red line with red stars) and energy loss (blue line with diamonds) as a function of the vertical offset in the dechirper for a gap of $6 \mathrm{~mm}$. The bunch charge is $200 \mathrm{pC}$.

To understand the reason for the difference between the wake potential calculated by our code and the single mode approach used for wakefield calculations in Ref. [2], we carried out calculations of shorter bunches for this dechirper to derive the Green's function. We found that the difference in wake field potentials came from the difference of Green's functions at small distances. This difference is important even for a bunch length of $0.67 \mathrm{~mm}$.

\section{CONCLUSION}

We have shown that the single mode approach does not accurately describe the wake fields of a short bunch in corrugated structures. We present an approximation of the Green's function in the analytical form for a corrugated dechirper. We calculate the wake potential for the PAL experiment [2] and found better agreement with the measured results than the single dominated mode assumption that was used to design the experiment. Based on our calculations, the single mode approximation [2-4] begins to break down when a bunch length becomes smaller than the period of the corrugated structure. In the next steps we plan to analyze the wake function for an offset trajectory in the dechirper.

\section{ACKNOWLEDGMENTS}

The author would like to thank Mike Sullivan for help and valuable comments, Axel Brachmann, Rick Iverson, Yunhai Cai, Paul J. Emma, Zhirong Huang and Franz-Josef Decker for support and interest in this work, and physicists of the Beam Physics Department for useful discussions. This work was supported by Department of Energy Contract No. DE-AC02-76SF00515.

[1] A. Novokhatski, F. J. Decker, H. Smith, and M. Sullivan, Wakefields in SLAC linac collimators, Phys. Rev. ST Accel. Beams 17, 124401 (2014).

[2] P. Emma et al., Experimental Demonstration of Energy Chirp Control in Relativistic Electron Bunches Using a Corrugated Pipe, Phys. Rev. Lett. 112, 034801 (2014).

[3] K. L. F. Bane and G. V. Stupakov, Impedance of a rectangular beam tube with small corrugations, Phys. Rev. ST Accel. Beams 6, 024401 (2003).

[4] Z. Zhang, K. Bane, Y. Ding, Z. Huang, R. Iverson, T. Maxwell, G. Stupakov, and L. Wang, Electron beam energy chirp control with a rectangular corrugated structure at the Linac Coherent Light Source, Phys. Rev. ST Accel. Beams 18, 010702 (2015).

[5] A. Novokhatski and A. Mosnier, in Proceedings of the Particle Accelerator Conference, BC, Canada, 1997 (IEEE, New York, 1997), p. 1661.

[6] A. Novokhatski and A. Mosnier, Report No. DAPNIA/ SEA-96-08, 1996.

[7] A. Novokhatski, A new Green's function for the wake potential calculation of the SLAC S-band constant gradient accelerating section, Nucl. Instrum. Methods Phys. Res., Sect. A 684, 46 (2012).

[8] A. Novokhatsky, Report No. SLAC-PUB-11556, 2005.

[9] A. Novokhatski, in Proceedings of the 31st International Free Electron Laser Conference (FELO9), Liverpool, UK (STFC Daresbury Laboratory, Warrington, 2009), p. 124401.

[10] A. Novokhatski, Field dynamics of coherent synchrotron radiation using a direct numerical solution of Maxwell's equations, Phys. Rev. ST Accel. Beams 14, 060707 (2011).

[11] V. A. Dolgashev, P. Emma, M. Dal Forno, A. Novokhatski, and S. Weathersby, in Proceedings of the workshop on Femto-second Electron Imaging and Spectroscopy (FEIS2), East Lansing, Michigan, 2015 (to be published) http:// www.feis-2.org. 